\title{
La scienza l'ignoranza la fede De sui ipsius et multorum ignorantia
}

\author{
Antonio Gagliardi
}

Università di Torino

\begin{abstract}
Per comprendere il senso del conflitto tra Francesco Petrarca e i quattro giovani averroisti in De sui ipsius et multorum ignorantia è necessario penetrare all'interno del linguaggio e mostrare la profondità della dottrina che vi si cela. A cominciare proprio dal quel termine, ignoranza, si attraversa tutta una biblioteca che rivela la consistenza vera della dottrina averroista. Per Averroè c'è un solo intelletto per tutta l'umanità e il bambino appena nato non ha un intelletto personale. Per questo motivo il bambino appena nato è simile all'animale senza ragione, il bruto. Questo intelletto si forma tramite l'acquisizione della scientia segnando l'acquisizione vera dell'umanità. L'uomo non nasce per natura ma per cultura e l'ignorante rimane nello stadio della brutalità. Tutta la vita dell'uomo deve essere dedicata all'acquisizione della scientia perché quando l'intelletto personale avrà acquisito tutti gli intelligibili si unirà con l'intelletto agente, la prima delle sostanze separate. Dopo gli sarà possibile giungere alla visione intellettuale di Dio. Questo è il fine ultimo, il sommo bene e la felicità. Si può comprendere il conflitto con il cristianesimo per il quale soltanto tramite Cristo e nell'altra vita è possibile giungere alla visione di Dio e alla beatitudine. Date queste coordinate si può comprendere come Petrarca cerca di rispondere da un punto di vista cristiano contrapponendo la fede alla scientia e a ogni via filosofica che porta a Dio. Nel conflitto con i giovani averroisti si rivela la pienezza di una frattura storica in antropologia ed escatologia e una via e della perfezione e della beatitudine incompatibile con il cristianesimo. Prendendo le difese del proprio cristianesimo come della propria scientia si sottrae alla filosofia il monopolio del sapere rivendicando una propria dignità intellettuale in sintonia con la fede cristiana.
\end{abstract}

Parole chiave: Petrarca, averroismo, ignorantia, fede, scienza.

\section{Abstract}

To understand the sense of conflict between Francesco Petrarch and the four Averroistic youths in De sui ipsius et multorum ignorantia, we must delve deep inside the language and illustrate the depths of the doctrine hidden within. Beginning exactly from this point, the search of an entire library reveals the true consistency of the Averroist doctrine. For Averroè, there is just one intellect for all humanity, of which the newborn child has no personal understanding. For this reason, the newborn child is similar to a senseless animal, or beast. This intellect is formed through the acquisition of scientia, marking the true acquisition of humanity. Man was not born by nature, but by culture, and the ignorant 
remain in the primate state. Man's entire life should be dedicated to the acquisition of scientia, because when personal intellect has acquired all knowledge, it will become united with agent intellect, the first of the separate substances. Thenceforth, it will be possible to see into the intellectual vision of God. This is the ultimate end, the greatest good, happiness. It is clear why a conflict exists with Christianity, which states that only through Christ and in another life is it possible to see the vision of God and to reach bliss. Based on this, it is understandable that Petrarch sought answers from a Christian point of view, setting aside his faith in scientia and in all philosophical paths leading to God. The conflict with the Averroist youths reveals the fullness of an historic fracture in anthropology and eschatology, a path, perfection and bliss that are inconsistent with Christianity. In the defence of Christianity, as with scientia, we attribute to philosophy the monopoly of knowledge, claiming a personal intellectual dignity in tune with the Christian faith.

Key words: Petrarch, averroism, ignorantia, science.

La difficile strada attraverso la scrittura del Petrarca per sondare le radici vere del contenzioso con i quattro giovani averroisti che nel De sui ipsius e multorum ignorantia lo hanno messo sotto accusa di ignoranza, ha bisogno di una preventiva ricostruzione del piano dottrinale e storico nel quale è riconoscibile un paradigma averroista. Non si tratta, infatti, di gelosie o invidie tra intellettuali ma della ragione più profonda che propone una diversa immagine dell'uomo e del suo destino mettendo in discussione le radici medesime del cristianesimo. Nel teatro della scrittura petrarchesca c'è la completa rappresentazione della crisi che la dottrina di Averroè induce nell'Occidente cristiano. Il modo in cui la lingua gestisce l'identità intellettuale del filosofo produce veli ed equivoci che soltanto risalendo alla biblioteca originaria possono essere dissolti. La gestione della scrittura è univoca e gli avversari sono costretti a parlare nel modo in cui viene loro permesso, entrando nella parola altrui sottostando all'altrui verità.

D'altra parte è pur vero che ormai da più di un secolo la dottrina del Commentatore è stata storicizzata e almeno da Tommaso d'Aquino in poi sono state disegnate le frontiere con il cristianesimo. Petrarca può usare un linguaggio allusivo perché gli interlocutori e il lettore sono dentro quel conflitto ed è sufficiente presentare la proposizione essenziale della dottrina per ottenere quell'effetto di verità nel quale ognuno può riconoscersi nel modo proprio. Per questo motivo, anche se la scrittura non è sistematica, è possibile scoprire il paradigma essenziale per il quale un averroista è identificabile. C’è un linguaggio che traccia la frontiera che divide un filosofo, seguace delle dottrine del Commentatore, e un cristiano. L'opera petrarchesca è dentro una storia e dentro un linguaggio e dell'una e dell'altro è possibile rendere conto.

Bisogna risalire sempre al modo in cui una biblioteca greco-araba, soprattutto nel XIII secolo, si sovrappone a quella cristiana creando un universo intellettuale parallelo espresso nella medesima lingua. 
[...] in filosofia e nelle scienze umane si verifica un coassiale spostamento di significati in alcune aree della cultura per parole tecniche già in uso.[...] Come dire che le parole rimangono le stesse dentro la comunicazione culturale, ma a certi livelli alti o non vogliono più dire le stesse cose o diventano polisemiche. ${ }^{1}$

Bisogna iniziare dal lessico. Cos'è l'ignoranza per un averroista? Perché, oltre l'autogratificazione polemica, è sufficiente il giudizio di ignoranza per procurare un tale allarme fino a coinvolgere tutta la configurazione intellettuale dell'individuo? Perché la fede cristiana si offre, sul piano individuale e collettivo, come antidoto alla pretesa dei giovani di giudicare qualcuno secondo virtù e conoscenza? E' necessario ricostruire il testo latente che sull'ignoranza propone un'immagine nuova, rispetto alla tradizione cristiana, dell'uomo e del suo destino. Petrarca e giovani parlano una lingua privilegiata, fatta di emblemi intellettuali sintetici in grado di significare oltre l'apparenza della parola. La restituzione di questi emblemi alla biblioteca originaria apre la dottrina averroista e il modo in cui è diventata storia collettiva portando allo scontro con la dottrina cristiana, con la sua immagine dell'uomo e con la sua escatologia.

L'ignoranza contiene tutto questo e, eseguendo il cammino delle parole, è possibile giungere nel luogo virtuale nel quale la scienza indica la via alternativa alla beatitudine rispetto a quella di Cristo. In quel luogo si incontrerà l'altro Petrarca, quello che in altri tempi ha tentato la medesima via che dalla scienza porta alla perfezione intellettuale e alla visione di Dio. Ora, dopo decenni di inquietudine intellettuale e religiosa, incontra se stesso dopo tutte le crisi e le soluzioni precarie e i compromessi parlando la stessa lingua dei giovani, rovesciandola contro le loro pretese di essere giudici. La costituzione del terreno comune mostra loro il comune sapere e la comune ascendenza filosofica. Ora può anche sembrare un ignorante ma c'è stato un tempo nel quale la strada della scienza segnava il medesimo cammino.

Si possono prendere due proposizioni de testo petrarchesco per segnare gli estremi della dottrina averroista e la strada che conduce l'uomo al fine massimo. L'uomo è posto tra l'ignoranza originaria e la visione di Dio, in questa vita, nella quale sta la beatitudine. Prima di tutto l'ignoranza.

Dicono che sono un uomo buono, anzi ottimo [...] Tuttavia chiamano illetterato e ignorante $[\ldots] .^{2}$

Da quale storia viene fuori la possibilità della conoscenza di Dio? Il fine massimo per il filosofo averroista consiste nella visione di Dio in questa vita.

Perché in questa vita Dio non può assolutamente essere conosciuto appieno, ma può essere amato con ardente devozione; e l'amore di Dio è in ogni

1. Maria CorTI, Scritti su Cavalcanti e Dante, Torino: Einaudi, 2003, p. 65-66.

2. Si cita da De sui ipsius et multorum ignorantia, in F. P. Opere latine, vol. II (a cura di Antonietta Bufano), Torino: UTET, 1975. 
caso un amore felice, mentre è talvolta fonte d'infelicità il conoscerlo, come accade ai demoni, che nell'Inferno tremano di fronte a lui, quando l'hanno conosciuto. ${ }^{3}$

Nessuno può conoscere Dio in questa vita. Tommaso d'Aquino ha scritto tutta la sua opera per dimostrare questo assunto. La visione di Dio è il fine dell'uomo sia per i filosofi sia per la dottrina cristiana. Differente è la via per giungervi. In questa vita per Averroè attraverso la scienza, nell'altra per il cristiano attraverso la grazia. In questa vita, però, c'è anche un modo di ascendere a Dio per il cristiano. La mistica ha da sempre posto una relazione diretta tra uomo e Dio. Petrarca contrappone alla via dei filosofi quella dell'amore secondo la tradizione agostiniana e poi francescana. Alla mistica averroista, ${ }^{4}$ totalmente intellettuale, si contrappone, almeno dall'Itinerarium mentis in Deum di Bonaventura da Bagnoreggio, una mistica della volontà e dell'amore.

Ma è necessario partire dalla definizione dell'ignoranza nell'antropologia averroista. La risposta bisogna cercarla nei commenti averroisti.

Ignorans est sciens in potentia [...] Et intendit per habitum formam scientie et per dispositionem contrariam ignorantiam. ${ }^{5}$

C'è un'implicazione reciproca tra la scienza e l'ignoranza e l'ignoranza è semplicemente la mancanza della scienza. In effetti, è necessario andare alla ricerca del significato di scienza e il modo in cui determina completamente l'antropologia e l'escatologia dell'uomo. E' necessario dare significato pieno anche a forma, formam scientie, perché proprio la sostanzialità (atto o perfezione) della scienza produce tutto il divenire dell'uomo fino a farlo ascendere all'unione con Dio.

T.27: Et scientia que est in actu est ipsa res[...]. Comm.: Et scientia que est in actu est ipsum scitum. ${ }^{6}$

La scienza è l'intelligibile estratto dall'ente e assimilato all'intelletto. L'intelletto privo di scienza, l'intelletto dell'ignorante, è un vuoto, una tabula rasa, secondo Aristotele. Ma c'è di più.

Per Averroè c'è un unico intelletto per tutta l'umanità e questo intelletto, possibile o materiale, è separato, non fa parte dell'anima umana. E' un'intelligenza caduta a contatto con la materia. Mentre l'anima sorge dalla materia e

3. Ibid., p. 1111.

4. Bruno NARDI, La mistica averrositica e Pico della Mirandola, in Saggi sull'aristotelismo padovano dal secolo XIV al secolo XVI, Firenze: Sansoni, 1958.

5. Averrois CoRdubensIs, "Commentarium magnum» in Aristotelis De anima libros, recensuit Francis Stuart Crawford, Cambridge, Massachussetts: The Mediaval Academy of America, 1953.

6. Ibid. 
muore con il corpo, l'intelletto è di sostanza spirituale ed immortale. La sua consistenza ontologica è simile a quella della materia prima, pura potenzialità che deve essere attuata dalla forma conosciuta. Per questo motivo l'intelligibile è sostanza. Si ha la tipica conformazione aristotelico-averroista tra materia e forma, tra potenza e atto.

Il bambino appena nato non ha un intelletto personale non essendo ancora la sua anima collegata, attraverso i fantasmi dell'immaginazione, con l'intelletto universale. L'intelletto personale si forma con l'acquisizione degli intelligibili. Questa dottrina è fondamentale quanto quella dell'intelletto unico. Se non si comprende la funzione dell'intelligibile, ovvero della scienza, nel determinare la nascita dell'intelletto personale viene meno la fondazione averroista. La conseguenza prima e più drammatica riguarda proprio il bambino appena nato. Per definizione l'uomo è l'animale dotato di intelletto. L'ignorante, l'uomo privo di scienza, non è uomo perché è anche privo di intelletto. Poiché è l'intelletto a fornire l'humanitas, la specificità umana rispetto alla genericità animale, è evidente che il bambino appena nato non è uomo. E' soltanto uomo in potenza poiché soltanto la scienza può farlo passare da animale a uomo facendogli formare l'intelletto.

Pertanto dire che il fanciullo intende in potenza può significare due cose: o che le forme immaginate in lui sono intelligibili in potenza o che l'intelletto materiale, atto per natura a ricevere l'intelligibile di quella forma immaginata, è ricettivo e congiunto a noi in potenza. [...] Dopo aver mostrato che l'intelletto materiale non ha alcuna delle forme materiali, [Aristotele] cominciò a definirlo dicendo che non ha altra natura se non la possibilità di ricevere le forme intelligibili materiali. [...] L'intelletto materiale è in potenza tutte le intenzioni delle forme materiali universali e non è in atto alcuno degli enti prima di intenderli. ${ }^{7}$

Se c'è soltanto un unico intelletto per tutta l'umanità e l'intelletto personale non è formato è chiaro che il neonato è simile al bruto, all'animale senza intelletto. Se è la scienza a trasformare il bruto in uomo, l'ignorante, l'uomo privo di scienza, è simile al bruto. L'ignoranza è lo stadio dell'animalità originaria prima di essere cancellata dall'acquisizione della scienza. Per questo motivo è necessario colmare la potenzialità dell'intelletto, raggiungere la perfezione intellettuale. Perfezione è parola chiave per indicare proprio il raggiungimento dello stadio ultimo, il completamente dell'intelletto per l'acquisizione di tutti gli intelligibili perché soltanto allora l'animale diventa totalmente uomo. Uomo si nasce per scienza e non per parto naturale. Uomo si diviene per cultura.

Per raggiungere la perfezione, la totale attuazione dell'intelletto, è necessaria tutta una vita perché è necessario trasformare tutto il potenziale conoscitivo. Per Averroè l'uomo deve acquisire tutti gli intelligibili, gli universali per raggiungere l'attuazione completa dell'intelletto. Deve conoscere tutto. 
Se è così, è necessario che l'uomo intenda mediante un intelletto proprio a sé tutti gli enti e che compia un'azione a sé propria in ogni ente, come intende mediante l'intelletto in abito tutti gli enti per intellezione propria, quando sia congiunto con le forme immaginative. ${ }^{8}$

Conoscere tutto diventa uno di quegli emblemi dottrinali che possono rappresentare tutta la dottrina averroista. Cosa succede quando l'intelletto ha raggiunto la perfezione? L'intelletto personale si unisce saldamente con l'intelletto agente, la prima delle sostanze separate, prive di materia. Questa è la felicità dell'uomo, secondo Averroè e secondo tutta la filosofia araba.

L'uomo, in questo modo, come afferma Temistio, è assimilato a Dio in quanto è tutti gli enti in qualche modo e in qualche modo li conosce; infatti gli enti non sono altro che la sua scienza, né la causa degli enti è altro che la sua scienza. Quanto mirabile è questo ordine, quanto straordinario è questo modo dell'essere! ${ }^{9}$

L'uomo diventa simile a Dio attraverso la scienza. Anche verbalmente si può comprendere come in Averroè si compie la promessa del serpente ad Eva: sarete simili a Dio. Tutta la storia biblico-cristiana viene cancellata da una nuova promessa di felicità e di divinizzazione per opera della scienza.

Il proemio alla Fisica diventa uno dei luoghi storici dell'immaginario filosofico perché offre l'estrema rappresentazione della mancanza di scienza. L'uomo privo di scienza, colui che non ha raggiunto la perfezione intellettuale, è simile al morto o alla statua di pietra. Il bruto e il morto come la statua di marmo diventano i simboli della mancanza di scienza in quanto mancanza di umanità.

[...]et declaratum est in scientia consyderante in operationibus voluntariis quod esse hominis secundum ultimam perfectionem ipsius, et substantia eius perfecta est ipsum esse perfectum per scientiam speculativam: et ista dispositio est sibi felicitas et sempiterna vita. Et in hac scientia manifestum est quod praedicatio nominis perfecti a scientia speculativa et non perfecti sive non habentis aptitudinem quod perfici possit est aequivoca, sicut nomen hominis quod praedicatur de homine vivo et de homine mortuo, sive praedicatio hominis de rationali et de lapideo. ${ }^{10}$

Anche qui le parole contengono un significato abissale se messe in relazione con la dottrina cristiana. Felicità e vita eterna sono il fine ultimo del cristiano redento da Cristo. Ora sono la promessa della filosofia.

Alla scienza è necessario aggiungere la virtù.

8. Cfr. A. Illuminati, op. cit., p. 168.

9. Ibid.

10. ARISTOTELIS, Opera cum Averrois commentariis, vol. IV, Edizione anastatica, Frankfurt am Main: Minerva, 1962. 
Et cum hoc consequitur cognitionem scientiae speculativae scientia de moralitate virtuosa, quoniam scientes istam scientia, cum erunt secundum ordinem naturalem, oportet eos de necessitate esse virtuosos in omnibus specibus virtutum moralium, quae sunt Iustitia, Temperantia, Fortitudo, Magnanimitas, Liberalitas, Veritas, Fiducia, Mansuetudo, et aliae de virtutibus hominun. ${ }^{11}$

Si compone in questo modo il binomio ulissiaco di virtù e conoscenza. Questo proemio alla Fisica diventa uno dei testi storici della linguistica averroista.

L'ultimo commento al nono libro della Metafisica mostra ancora, in forma negativa, la condizione dell'uomo privo di scienza.

Et hoc intendebat cum dicit: intelligere eas non est verum idest cum dicimus quod apud nos non est verum de substantiis simplicibus, non intendimus quod accidit nobis error, qui est ignorantia secundum habitum, sed accidit privatio veritatis quae est ignorantia tantum et hoc est in virtute rationali sicut caecitas in oculo. Et innuit per hoc quod non intelligere res separatas per intellectum humanum est in intellectu nostro in primo simile caecitati in oculo antequam perficiatur intellectus. ${ }^{12}$

L'uomo che non giunge alla conoscenza delle sostanze separate per colpa dell'ignoranza è simile al cieco. Soltanto la perfezione intellettuale, l'attuazione totale dell'intelletto, permette di raggiungere quel fine.

Nel primo commento al secondo libro della Metafisica appare il fine massimo, la conoscenza della verità in quanto conoscenza di Dio.

[...]quia comprehensio veritatis non est impossibilis in multis rebus credimus enim necessario nos scire veritatem in multis rebus. [...]. Et, quia dispositio intellectus de re intelligibili est sicut dispositio sensus de re sensibili, assimilavit virtutem intellectus in comprehendendo intellecta abstracta a materia modo debilissimo visui in sentiendo, sicut vespertilionis, non comprehendendo maximum sensibilium, sicut Solem. Sed hoc non demostrat res abstractas intelligere esse impossibile nobis: sicut inspicere Solem est impossibile vespertilioni quia fecit illud quod est in se naturaliter intelligibilem non intellectum ab alio, sicut si fecisset Solem non comprehensum ab aliquo visu.

Il pipistrello (o il gufo) dinanzi al sole diventa il simbolo dell'intelletto umano dinanzi a Dio. L'uomo può conoscere Dio come il pipistrello può guardare la luce del sole in modo molto debole.

Questo è il piano testuale minimo nel quale si mostra il cammino del bruto a uomo perfetto fino a poter guardare il sole Dio. Tommaso d'Aquino è il primo antagonista che confuta organicamente la dottrina di Averroè specialmente nella Contra gentiles. Questa è la sua posizione sul bruto. 
Dunque l'uomo, pur avendo l'intelligenza, non differirebbe dagli animali bruti. [...] Ora, il bambino, anche prima di uscire dal seno materno, appartiene alla specie umana; eppure in lui non ci sono fantasmi che siano intelligibili in atti. Dunque l'uomo non è dotato d'intelletto per il fatto che l'intelletto si unisce all'uomo mediante le specie intelligibili esistenti nei fantasmi. ${ }^{13}$

L'uomo è tale fin dal seno materno. Nello stesso modo viene negata la possibilità di conoscere Dio.

Ora, se in questa vita non possiamo avere l'intellezione delle sostanze separate, per la connaturalità del nostro intelletto con i fantasmi, meno che mai potremo vedere l'essenza divina, la quale trascende tutte le sostanze separate. ${ }^{14}$

Il cammino del bruto, in compagnia della scienza, porta alla visione di Dio. Una escatologia filosofica si sovrappone a quella cristiana e offre gli strumenti per giungere al fine senza passare attraverso la fede e la chiesa. Una situazione analoga si è già prodotta nell'Islam e il conflitto tra i filosofi e gli uomini del libro divino, teologi ortodossi o mistici sufi, esibisce l'inconciliabilità tra la via della scienza e quella delle fede. Averroè introduce questo conflitto nell'Occidente cristiano e l'Occidente cristiano reagisce allo stesso modo, con le medesime coordinate dottrinali, pur nella specificità cristiana. Un averroista si riconosce da queste due proposizioni dottrinali, la perfezione e la felicità, organizzate come un unico viaggio di conoscenza che porta il filosofo direttamente al cospetto di Dio. Dalla seconda metà del Duecento è frequente l'immagine del bruto o dell'uomo privo di perfezione quale limite della condizione umana. La coscienza di un limite contiene già in sé l'alternativa poiché quella negatività può essere e deve essere superata. Il bruto è l'apocalisse dell'uomo ed è figlio dell'ignoranza. La scienza lo porta alla pienezza dell'umanità e lo eleva al divino.

Abbracciando la totalità del sapere, possedendo ogni virtù intellettuale e morale, il filosofo rappresentava il massimo della perfezione raggiungibile sulla terra. Innalzandosi fino alla visione degli enti metafisici più elevati —le sostanze separate e Dio - egli portava infatti a piena realizzazione ciò che vi è di più specificamente umano, la razionalità. [...] Chi non è filosofo — scriveva senza mezzi termini Alberico di Reims - «non est homo nisi equivoce»; il sommo bene cui un mortale può e deve aspirare — chiariva Boezio di Dacia- consiste nel piacere ricavabile dall'esercizio della virtù e dalla conoscenza del vero, e chiunque non lo raggiunga «non habet rectam vitam» e deve sapere di essere «imperfectum individuum in specie sua, nec habet actiones humanas». Quanti rinunciano a realizzarsi intellettualmente - faceva eco Giacomo di Douai nella chiusa delle sue inedite questioni sul De anima — non si distinguono dai

13. San Tommaso D'Aquino, Somma contro i gentili (a cura di Tito S. Centi), Torino: UTET, 1975, p. 405. Per il rapporto tra Tommaso d'Aquino e Averroè, cfr. Antonio GaGLIARDI, Tommaso d'Aquino e Averroè. La visione di Dio, Soveria Mannelli: Rubbettino, 2002.

14. Ibid., p. 654. 
bruti, e non meritano pienamente la definizione di uomini, essendo tali solo in potenza $[\ldots] .{ }^{15}$

Il modo in cui Dante all'inizio del Convivio pone il rapporto tra l'ultima perfezione e l'ultima felicità è pienamente averroista anche se, nel corso dell'opera, cerca di eliminare l'ascesa alla visione di Dio.

[...] onde, acciò che la scienza è ultima perfezione de la nostra anima, ne la quale sta la nostra ultima felicitade, tutti naturalmente al suo desiderio semo subietti.

E' la scienza a portare l'uomo alla perfezione ultima e all'ultima felicità, il sommo bene o beatitudine. Può essere accettato questo da un cristiano? Non è Cristo la via alla beatitudine dell'uomo ottenibile dopo questa vita? Dante qui sicuramente non è tomista. Soltanto nella Commedia la ricerca di una sintesi efficace tra il cammino della scienza e quello della grazia e dell'illuminazione divina permetterà di portare Tommaso dentro il proprio progetto di conciliazione universale conciliandolo con Sigieri di Brabante.

Non si tratta di un generico contrasto tra ragione e fede. Qui è in discussione l'alternativa radicale tra la scienza e Cristo per raggiungere lo stesso fine, la visione di Dio e la beatitudine. Senza questa alternativa non si comprende l'inconciliabilità tra cristianesimo e averroismo. La scienza cancella Cristo dall'escatologia della beatitudine. Si può ricorrere sempre a Dante, questa volta nella Commedia, per il senso di questa alternativa.

Matto è chi spera che nostra ragione

possa trascorrer la infinita via

che tiene una sostanza in tre persone.

State contenti, umana gente al quia;

ché, se potuto aveste veder tutto,

mestier non era parturir Maria;

Purgatorio, III, 34-39

Il conoscere/vedere tutto, la perfezione intellettuale nell'acquisizione di tutti gli intelligibili, non può permettere il viaggio dell'uomo a Dio. E' necessario Cristo, il parto di Maria. Il viaggio tragico di Ulisse dalla condizione di bruto all'oltrepassamento del confine tra l'umano e divino si conclude con la condanna. Il nuovo Ulisse, Dante, mostra come senza la grazia di Dio e la mediazione di Maria non è possibile giungere al vertice dell'universo dove sta il Dio dei filosofi e quello cristiano.

Anche se in forma sintetica questo è il paradigma averroista nella dottrina e nella forma storica. La scrittura di Petrarca è solidamente fondata su questi 
assunti e la lingua, direttamente o indirettamente, tiene conto delle dottrine originarie. Una coscienza cristiana pone una frontiera tra il cammino dell'uomo a Dio secondo la via dei filosofi e quella segnata da Cristo. Ora è possibile ritornare al testo originario ed esaminare il modo in cui gli antagonismi vengono fissati. Il contenzioso si allarga a tutti gli altri problemi che la dottrina averroista pone alla coscienza intellettuale e religiosa contemporanea, dall'eternità del mondo alla poesia. Si ha un quadro veramente completo di una situazione storica nella quale la filosofia sta disegnando una nuova condizione dell'uomo. Evidentemente il punto di vista rimane quello dell'autore e i quattro malcapitati non hanno alcuna possibilità di far valere le proprie ragioni.

Sul problema della scienza e dell'ignoranza è evidente che si parlano due lingue diverse e reciprocamente estranee. Quell'idea di scienza, di matrice averroista, si realizza in un sapere enciclopedico su tutti gli enti di natura. La scienza è prima di tutto conoscenza della natura. L'ironia sul sapere inutile o errato fa parte di questa polemica. L'unicità dell'ignoranza produce confusione e diventa il terreno di scontro tra due atteggiamenti intellettuali. La scienza dei filosofi prefigura un sapere tecnico, organizzato razionalmente, ordinato secondo categorie. Dall'altra parte c'è la conoscenza umanistica della quale la retorica diventa la lingua ufficiale. Siamo già all'interno di quella rottura che conferisce all'Occidente uno dei suoi aspetti fondamentali, tra lettere umane e scienza. Viene meno in questo modo la pretesa che la scienza possa portare alla felicità, sia vera o meno. L'irrisione della scienza, nelle sue forme più evanescenti, diventa critica della pretesa ultima di raggiungere la felicità.

Tutte queste nozioni [...] anche ammesso che rispondessero a verità, non contribuirebbero per nulla alla nostra felicità. ${ }^{16}$

In questo modo viene sottratto ai filosofi anche il sapere ultimo, la conoscenza delle origini e dei fini dell'uomo.

A che può servire, di grazia, conoscere le particolarità delle belve, degli uccelli, dei pesci, dei serpenti e ignorare, invece, e disprezzare la natura umana, lo scopo della nostra nascita, da dove veniamo e dove andiamo ${ }^{17}$

L'escatologia averroista viene cancellata assieme alla scienza che la produce. Il sapere sull'uomo diventa esclusivo appannaggio della religione perché portatrice di una escatologia totalizzante nella quale soltanto c'è l'origine e il fine dell'uomo. Il conflitto tra religione e cultura ha prodotto ormai una frattura insanabile. L'avere oscurato il fine del filosofo mostra questo bisogno di antagonismo senza compromesso sui fini ultimi. La religione agli ignoranti e la scienza ai filosofi. Si tratta di una delle forme storiche dell'averroismo. 
In effetti, per gente come loro, che va tronfia di far la figura dell'intellettuale, non vi è cosa più spregevole della devozione religiosa $[\ldots] .{ }^{18}$

L'ignoranza, come imperfezione, può essere risanata soltanto dopo la morte, per il cristiano. La morte diventa via necessaria alla beatitudine. Soltanto dopo la morte l'uomo può vedere Dio.

Ma frattanto, finché non giunge la fine di questo esilio, con la quale avrà termine questa nostra imperfezione, mi consolo — nei limiti della conoscenza che per ora ci è concessa- considerando la natura a tutti comune. ${ }^{19}$

L'alternativa con i filosofi è sottintesa nel porre in vita il raggiungimento della perfezione e della felicità. In effetti è immediata la constatazione della loro presunzione nel giudicarsi degni della conoscenza angelica e della felicità connessa.

Beati nel loro errore i miei giudici, che non hanno bisogno di simile conforto, beati dico, non per il sapere, ma per l'assenza di errore e di presuntuosa ignoranza coloro che ritengono non manchi per la conoscenza delle cose angeliche, mentre indubbiamente per quella della realtà umana manca molto a tutti e a molti tutto addirittura. ${ }^{20}$

Che per scienza angelica si debba intendere la conoscenza che hanno gli angeli, prima di tutto la loro conoscenza di Dio si può comprendere in Tommaso d'Aquino. Indirettamente Petrarca attribuisce ancora ai giovani averroisti la pretesa di giungere alla conoscenza di Dio tramite la scienza, la conoscenza del tutto.

Nello stesso modo, con una sottile ironia, la richiesta di una lunga vita. In effetti per giungere alla perfezione una lunga vita è necessaria e si può ottenere soltanto verso la fine di essa, come afferma Averroè (commento 36).

Ahimè, amico mio, quali sciagure non comporta una vita che si protrae troppo a lungo? ${ }^{21}$

Una vita lunga non capita a tutti e può anche essere piena di infortuni. Chi può assicurare la felicità? Viene meno per la maggior parte degli uomini la possibilità di attingere la perfezione e la felicità. Anche se sommerso nel teatro della parola petrachesca il testo filosofico originario continua a produrre senso in stato di latenza. Così è possibile far cadere Aristotele nell'ignoranza. 
Per conto mio, credo che Aristotele fu bensì una personalità di grande rilievo e di molta dottrina, ma che era un uomo e perciò ritengo che egli potesse ignorare alcune cose, anzi molte. ${ }^{22}$

Il problema della felicità, come viene trattato da Aristotele all'inizio e alla fine dell'Etica Nicomachea diventa il termine di confronto vero con quella cristiana. Non spetta alla filosofia trattare della felicità vera.

[...] egli a tal punto ignorava la vera felicità, che nella conoscenza di essa potrebbero essere non dico più sottili, ma più felici una qualsiasi vecchietta devota o un pescatore, un pastore, un contadino che abbiano fede in Dio.[...] ho l'impressione che lui abbia visto la felicità come la nottola vede il sole, che cioè ne abbia visti la luce e i raggi ma non l'essenza [...] e non ha capito, o se li ha capiti ha trascurato quei princìpi, senza i quali la felicità non può assolutamente esistere, ossia la fede e l'immortalità. ${ }^{23}$

Dentro Aristotele c'è Averroè. L'allusione al primo testo e commento del secondo libro della Metafisica, con il simbolo del gufo o pipistrello, rovescia la visione del filosofi in cecità. La metafora o l'allusione sono in grado di rappresentare il modo in cui il filosofo, superbo, pretende di giungere alla conoscenza suprema attraverso la scienza e in quanto scienza.

Costoro cercano di captare con la loro superba millanteria i segreti della natura e gli arcani ancor più profondi di Dio, che noi accettiamo con l'umiltà della fede; e non li captano, né vi si accostano neppure, ma, pazzi come sono, ritengono di esserci arrivati e di tenere in pugno il cielo[...]. ${ }^{24}$

Per tenere in pugno il cielo e conoscere i secreti di Dio è necessario giungere fino in cielo e fino a Dio. Le autorità citate fanno parte del corredo storico di confutazione della pretesa dei filosofi. Anche la citazione omerica serve a tenere lontano lo sguardo del filosofo da Dio. Non soltanto gli uomini non possono vedere Giove ma neanche Giunone sua sorella e moglie.

[...] in Omero Giove diffida con gravi minacce dall'osar di scrutare nel suo intimo segreto o dal presumere che esso si possa conoscere, non un uomo mortale né un qualunque dio, ma la potente Giunone, sua consorte e regina degli dei. ${ }^{25}$

Si può conoscere e studiare ma senza pretendere di oltrepassare i limiti dell'umano. L'atto di umiltà personale iscrive l'autore in una prospettiva cristiana aliena da ogni curiosità oltraggiosa. 
[...] ringrazio Iddio, di avermi dato questo intelletto, per inetto e modesto che sia, e uno spirito, per niente irrequieto, che non va alla ricerca di cose più grandi di lui né soffre della curiosità d'indagare su cose che sono difficili da scoprire e micidiali una volta scoperte; ma quanto più sento parlare contro di Cristo, tanto più amo Cristo e mi rafforzo nella fede di Cristo. ${ }^{26}$

Chi parla non farà mai la fine di Ulisse. La scienza e la fede, lo spirito ulissiaco e quello cristiano, sono in campo per determinare una frontiera di comune incompatibilità. Cicerone diventa il velo dietro il quale è possibile continuare a parlare del presente su argomenti scottanti sul piano teologico.

Senza dubbio, conoscere Dio, non gli dèi, questa soltanto è vera e altissima filosofia; a condizione, intendo, che alla conoscenza si associ le fede e la pratica religiosa. ${ }^{27}$

Cosa è successo? La metafisica aristotelico-averroista sembra riportare l'uomo al tempo dei pagani con una pluralità di dei mettendo sullo stesso piano Dio e le altre intelligenze celesti, le sostanze separate. La conoscenza delle intelligenze celesti, fra la quali Dio è soltanto la prima, quale fine del cammino del filosofo, omologa sostanzialmente il conoscente e il conosciuto, l'uomo e Dio. L'intelletto umano viene considerato della medesima natura di Dio, anche se di perfezione inferiore. La lunga digressione sulla natura di Dio mostra come si è costituito un nuovo Olimpo nel quale stanno tutte le intelligenze e al quale può aspirare anche l'uomo. Ora è necessario affermare l'assoluta trascendenza di Dio. Non solo: Dio è creatore e governa l'universo con la sua volontà provvidenziale.

[...] tutto quanto vediamo con gli occhi o percepiamo con l'intelletto è stato creato per il bene degli uomini, è di origine divina ed è governato dal volere provvidenziale di Dio. ${ }^{28}$

C'è un unico Dio che governa l'universo e non una pluralità di intelligenze, come vuole Averroè. Cicerone, in predicato di diventare cristiano, ora è costretto a diventare averroista per sostenere la polemica di Petrarca sulla costituzione molteplice dell'olimpo dei filosofi.

Chi sono dunque codesti nuovi, moderni, infami dèi che cerchi di far entrare a forza nella casa del Signore? [...] Così, tutto d'un tratto, lo hai mescolato a creature ribelli e a spiriti immondi? [...] E come se non bastasse, fai diventare sensibili, animati, e — stoltezza somma - altrettanti dèi, il sole, la luna, le stelle e infine persino questo mondo percettibile, che noi vediamo e tocchiamo, su cui posiamo i piedi. ${ }^{29}$

26. Ibid., p. 1069.

27. Ibid., p. 1071.

28. Ibid., p. 1083.

29. Ibid., p. 1085. 
Il riferimento ai cieli, con le loro intelligenze, nel nome e nella funzione ricorda la molteplicità di dei dell'olimpo pagano, quasi un ritorno al tempo precristiano.

Ora Petrarca scende in campo di persona per confutare la colpa dell'ignoranza mostrando che si tratta soltanto della propria fede cristiana.

Se il pericolo di un castigo non li minaccia e non hanno testimoni, confutano la verità e la religione, nascondendosi in angoli riposti a deridere Cristo e ad adorare Aristotele che non capiscono [...]. ${ }^{30}$

Una confusa testimonianza di tribunali e persecuzioni nei confronti dei filosofi si intravede in queste parole. Non tutti hanno la possibilità di professare le proprie idee. Anche Tommaso d'Aquino, nel De unitate intellectus contra averroistas, aveva accusato Sigieri di Brabante di nascondersi negli angoli.

Che se taluno, gloriandosi d'una falsa scienza, ha qualcosa da dire contro quanto abbiamo scritto, non parli negli angoli né davanti a fanciulli che non sanno giudicare di questioni sì ardue, ma impugni questo scritto, se ne ha l'ardire, e troverà non solo me, che sono il minimo di tutti, ma molti altri cultori della verità, i quali si opporranno al suo errore, o provvederanno a smascherarne l'ignoranza. ${ }^{31}$

Si può vedere come anche in questo breve testo scienza ed ignoranza sono in antitesi e pongono l'alternativa secca all'interno del mondo dei filosofi. La lingua dice già molto e sia Tommaso d'Aquino sia Petrarca la gestiscono polemicamente. L'antitesi tra Cristo e Aristotele racchiude tutti gli antagonismi e i conflitti tra l'atteggiamento del filosofo e quello del cristiano. Con questa lingua e con questi antagonismi possiamo guardare dentro la storia il modo in cui un nuovo intellettuale non si riconosce più nel cristianesimo. L'attestazione è importante perché, al di la delle polemiche personali, è riconoscibile una misura laica fondata sulla conoscenza filosofica.

Si apre una finestra sulla condizione vera del filosofo, ormai costretto a nascondersi e non poter parlare liberamente. Una situazione simile si intravede in una frase successiva, uno dei luoghi comuni della cosiddetta doppia verità.

[...] poiché manca loro il coraggio di sputar fuori i propri errori, hanno l'abitudine di dichiarare formalmente che al momento discutono lasciando del tutto da parte la fede. ${ }^{32}$

30. Ibid., p. 1093.

31. Tommaso D'AQUINo, Trattato sull'unità dell'intelletto contro gli averroisti (a cura di Bruno Nardi), Spoleto: Centro italiano di Studi sull'alto Medioevo, 1998, p. 196-197.

32. Cfr. Antonietta Bufano, cit., p. 1095. 
Si tratta soltanto della doppiezza della parola costretta a dissimulare nel gioco delle apparenze una verità innominabile pubblicamente. Si può discutere di problemi così alti sospendendo il giudizio secondo la fede? Per il cristiano evidentemente no. Sull'eternità del mondo, uno dei maggiori errori della filosofia secondo la teologia cristiana, Petrarca è molto informato. ${ }^{33}$ Il tema che più di ogni altro mette in discussione la teologia della creazione è al centro del dibattito contemporaneo.

Il cammino all'interno della scrittura petrachesca, alla ricerca dei nuclei dottrinali, riporta ancora il senso della perfezione intellettuale e i mezzi per raggiungerla: la conoscenza di tutti gli intelligibili nella conoscenza di tutte le cose (rerum omnium scientiam).

A me sembra invece proprio il contrario; e non sono disposto ad ammettere che un qualsiasi uomo abbia potuto raggiungere con mezzi umani un sapere universale. $^{34}$

L'uomo non può conoscere tutto. Non solo. I filosofi hanno detto molte menzogne.

Se così è, essi sanno che i filosofi hanno detto molte menzogne[...]. ${ }^{35}$

Petrarca rovescia sui filosofi il detto di Aristotele sui poeti: Poetaeque, secundum proverbium, multa mentiuntur. Questa frase diventa il momento genetico del conflitto tra poeti e filosofi su un'autorità in sintonia con tutta la filosofia del tempo. ${ }^{36}$

Poi si ritorna sul tema fondamentale, la conoscenza di Dio. Il binomio di virtù e conoscenza disegna anche le frontiere sulle quali si attesta la scienza dell'uomo.

Perciò sono in grave errore quelli che impiegano il loro tempo nel cercar di conoscere la virtù, non di farla propria, e in errore gravissimo quanti lo impiegano a conoscere Dio, non ad amarlo. ${ }^{37}$

Una scienza di Dio quale meta ultima dell'uomo non è possibile. Soltanto l'amore, secondo la tradizione agostiniana e francescana, può raggiungere veramente Dio. Il rapporto tra conoscenza e amore ormai è stabilito da una lunga polemica tra i francescani e i tomisti e Petrarca lo ripropone mediando amore e conoscenza. Non si può amare ciò che è totalmente sconosciuto.

33. Enrico FEnZI, «Petrarca e l'eternità del mondo. Appunti per un commento al De ignorantia», Intersezioni, 1994.

34. Cfr. Antonietta Bufano, cit., p. 1103.

35. Ibid., p. 1105.

36. Antonio Gagliard, Giovanni Boccaccio. Poeta filosofo averroista, Soveria Mannelli: Rubbettino, 1999, cap. II.

37. Cfr. A. Bufano, op. cit., p. 1111. 
E quantunque non si amino le cose completamente sconosciute, è sufficiente tuttavia conoscere Dio e la virtù entro i limiti oltre ai quali non è dato spingersi $[\ldots] .^{38}$

Si può vedere la posizione analoga di Tommaso d'Aquino nella Contra Gentiles. «Ora questa è l'intellezione: poiché non è possibile volere ciò che ancora non si conosce». (III, XXVI). In ogni modo, nella conoscenza umana, non si devono superare la frontiere poste tra l'uomo e Dio, le colonne d'Ercole che nessuna scienza può infrangere. A questa condizioni è anche possibile trovare un accordo con i filosofi.

E' necessario superare il dualismo di scienza e fede dei filosofi, eliminando la loro irreligiosità. Se la religione nasce dall'insicurezza e la scienza si propone come depositaria delle verità ultime, è evidente che non è possibile alcun accordo. Al di là del nominalismo c'è un bisogno profondo di unificare scienza e fede nel modo in cui un cristiano può certificare.

[...] la conoscenza della vera fede, invece, è la più profonda, la più sicura e infine la più felice di tutte le scienze. ${ }^{39}$

Soltanto la consapevolezza della pochezza della conoscenza umana può permettere di accostarsi alla conoscenza divina.

Che vi è d'altronde, in quanto è successo, che possa tormentare profondamente un animo nobile, che conosce le cose umane e anela a quelle celesti, quando misura la propria piccolezza e riflette su quanto essa sia vicina al nulla? ${ }^{40}$

L'uomo deve prendere atto che non può conoscere tutto e che soltanto attraverso la rivelazione divina c'è vera conoscenza. Ippia contemporaneamente nasconde Averroè e lo confuta.

Ma poiché ormai è cosa certa che gli uomini non sanno tutto, anzi neppure molto, ed è noto — sconfessata e ripudiata ormai da tempo l'Accademia - che si può conoscere qualcosa solo attraverso la rivelazione divina, accontentiamoci di sapere quel tanto che basta alla salute eterna. ${ }^{41}$

La conoscenza del limite e di ciò che è necessario per ottenere la salvezza eterna chiude di fatto il cammino nella scienza e nell'ignoranza. C'è sempre una memoria ulissiaca del limite a porre l'uomo dinanzi alle proprie responsabilità di cristiano. Non tutto il filosofo si può ritrovare nel cristiano. Soltanto questa consapevolezza ultima può permettere di sostituire la scienza con la fede quando è la certezza della beatitudine eterna a muovere il cammino dell'uomo e non quella felicità momentanea promessa dalla scienza nella visione di Dio in vita. 
Petrarca, nella dissimulazione della propria ignoranza, espone tutta la biblioteca personale dimostrando di quale scienza può disporre. Non è lui l'ignorante e il bruto. Chi può esibire una tale biblioteca non può essere un ignorante con tutte le conseguenze del significato latente. I giovani vengono sommersi nella sua erudizione.

Vi sono tante accezioni di scienza e questa degli averroisti è una novità che produce un'onda sismica nella coscienza individuale e nella cultura collettiva. C'è un'idea di scienza, connessa a un fine di perfezione e felicità. Con questo mito della scienza è necessario fare i conti fino in fondo perché in grado di sedurre le coscienze e rifondare la cultura occidentale. Il mito dell'uomo che nasce bruto e diventa Dio, per scienza e virtù, sta alla base di ogni altro mito letterario e intellettuale. Si può dire, con un linguaggio più moderno, che la scienza determina il progresso dello spirito umano. Il mito di Ulisse è disperso nelle scritture averroiste e soltanto la poesia è in grado di ricostruirne l'immagine unificando le sparse tessere. Cosi diventa chiave ermeneutica per chi tenta il guado avventuroso delle colonne d'Ercole come di chi cerca di trattenere la propria barca prima del confine vietato.

Quest'opera di Petrarca permette di formulare un quadro storico e organico della complessa situazione intellettuale prodotta dalle dottrine averroiste. Pur nell'opacità della scrittura appare il motivo di fondo di due universi in conflitto, filosofia e cristianesimo, perché concorrenti sul piano dei fini ultimi, la perfezione e la felicità. Tutto il resto è accessorio. Scienza e fede, con la negatività originaria dell'ignoranza, stanno sulla frontiera dell'umano e del divino e regolare l'accesso alla conoscenza di Dio. Due modi di salire al cielo e godere della beatitudine divina sono possibili attraverso Cristo o la scienza.

Poi è possibile leggere il Canzoniere per scoprire come, in un'altra lingua e in altri tempi, tutto è già stato detto per confermare un'inquietudine personale e la crisi della stagione averroista di Petrarca. Basta mettere in evidenza l'alternativa tra Cristo e la scienza di nome Laura nel costruire la strada che porta al cielo. ${ }^{42}$

Tanto mi piacque prima il dolce lume ch'i passai con diletto assai gran poggi per poter appressar gli amati rami: ora la vita breve e 'l loco e 'l tempo mostrammi altro sentier di gire al cielo et di far frutto, non pur fior' et frondi. Altr'amor, altre frondi et altro lume, altro salir al cielo per altri poggi cerco, ché n’è ben tempo, et altri rami.

42. Antonio Gagliard, Gunizzelli Dante Petrarca. L'inquietudine del poeta, Alessandria: Edizioni dell'Orso, 2003. 
L'insistenza di «altro» mette in luce il dramma del filosofo e del cristiano. Lalloro e il legno della croce sono in reciproca incompatibilità come la luce di lei e quella della grazia. Petrarca ha abbandonato la via che conduce a cielo disegnata dall'alloro. Laura ha conteso a Cristo la via per salire al cielo e la radicale alternativa tra i due appare in tutta la sua inconciliabilità. Il «far frutto", la perfezione cristiana è altra cosa rispetto a quella filosofica. La scienza ha fatto venire meno il monopolio del divino detenuto dal cristianesimo come ha fatto venire meno l'unicità della beatitudine. Com'è possibile a una donna tutto ciò? I miti intellettuali comuni diventano miti esistenziali personali. La scienza di nome Laura o Beatrice, o semplicemente la donna mia, seduce e fa innamorare di sé. Poi sparisce alla vista e si fa cercare producendo soltanto dolore e sofferenza nel suo amante. Soltanto alla fine del lungo cammino di perfezione si offre come mercede intellettuale indicando la strada che conduce fino a Dio.

Ma questa strada, per un cristiano, porta sempre allo scontro con il Dio cristiano. Petrarca Ulisse lo ha sperimentato di persona e il suo "picciol legno» più volte ha rischiato il naufragio spinto dall' "aura soave». Due modi di raggiungere il porto, la beatitudine della visione di Dio, si contendono la strada. Soltanto l'intervento divino, "poi piacque a lui», salva dall'altro intervento che condanna, "com'altrui piacque».
L'aura soave a cui governo et vela commisi entrando a l'amorosa vita e sperando venire a miglior porto, poi mi condusse in più di mille scogli; et la cagion' del mio doglioso fine non pur d'intorno avea, ma dentro al legno.
Chiuso gran tempo in questo cieco legno errai, senza levar occhio alla vela ch'anzi al mio dì mi trasportava al fine; poi piacque a lui che mi produsse in vita chiamarme tanto indietro da li scogli ch'almen da lunge m'apparisse il porto.

Può veramente l'amore carnale per una donna portare a esiti così tragici per la salvezza eterna? La seduzione di una donna mortale può avere tanto potere da condurre alla perdizione eterna? Dall'altra parte sembra possedere un potere demoniaco, da anticristo, nel costruire un itinerario antagonista alla beatitudine cristiana. Con una donna si pensa sempre al solito peccato. Invece è il peccato di Ulisse, attraversare il confine vietato tra l'umano e il divino, a condurre a una possibile dannazione. E' sempre l'amore per questa donna dai poteri assoluti a trascinare per mari ignoti e per ascese celesti. L'epistola sull'ascesa al monte Ventoso conferma, nell'allegoria personale, che vi sono due modi di salire su per i monti come per salire in cielo. 
Da tutta la tradizione poetica, a cominciare dai Siciliani, è evidente l'identità di amore e conoscenza. La via dell'amore è la stessa della scienza, attraverso le facoltà dell'anima, dagli occhi all'intelletto, passando per l'immaginazione. Poi la scienza apre la strada che porta al cielo. Dovrebbe essere chiaro che tra la via che porta al cielo e il naufragio procurato da Laura c'è un nesso indissolubile. Mentre è interdetto il passaggio delle colonne d'Ercole sulla terra è possibile quello tra la terra e il cielo? Non ci sono le colonne d'Ercole tra la terra e il cielo? Le metafore e le allegorie hanno una loro necessità imposta dalla dottrina che le regge. Soltanto se c'è la possibilità di oltrepassare il velo dell'immaginazione poetica e giungere ai fondamenti intellettuali vengono fuori tutte trame di una storia che nell'autobiografia di Petrarca si realizza in tutta la sua drammaticità in un conflitto indissolubile tra la forma della scrittura e quella della coscienza.

L'amore per la scienza di nome Laura ha fatto errare per un mare in tempesta, presagendo nel proprio «cieco legno» il «cieco carcere» infernale. L'«altezza d'ingegno", in ogni modo, porta lontano dalla via della salvezza. Un «dolce lume» connette anche il sonetto precedente con il medesimo canto dantesco: «non fiere li occhi suoi lo dolce lume?» (Inferno, X, 69). La salvezza viene dallo stesso Dio che era stato sfidato sul piano dell'ultima felicità, il porto. L'approdo finale nel quale c'è perfezione intellettuale e beatitudine secondo la promessa dei filosofi e quella cristiana. Su questa alternativa è possibile seguire tutta la crisi di Petrarca nel Secretum e nel Canzoniere. La via della scienza alla visione di Dio, dall'altezza della presente opera, appare lontana, frutto soltanto di una biblioteca molteplice in grado di confutare l'errore altrui. Poi tutto diventa un'azione drammatica quando il poeta, dopo l'esperienza della secessione e del conflitto insanabile, cerca un equilibrio tra le nuove istanze umanistiche e la propria coscienza cristiana.

C'è sempre da mettere nel conto di queste crisi e dei loro risvolti filosofici e religiosi una fondazione nella coscienza personale che permette di ricostruire il proprio itinerario intellettuale tra le antitesi e conflitti. Non c'è dubbio che anche questa coscienza di sé è frutto della scienza. L'autocoscienza intellettuale è direttamente proporzionale alla scienza acquisita. Il vero miracolo di questo tempo storico è la nascita della coscienza intellettuale, una coscienza laica che viene in aiuto anche quando si tratta di sorreggere una crisi che mette in discussione la fondazione religiosa. Il conflitto tra coscienza intellettuale e coscienza religiosa non diventa apocalittico quando la scienza offre quel sostegno in grado di trovare un'identità forte.

La coscienza di sé è in stretta relazione con la scienza acquisita e chi può fruire di una biblioteca ampia e articolata può anche mostrare la profonda consapevolezza dell'io intellettuale. Riflettere su se stessi porta a compimento la ragione della crisi e della critica e la scienza dell'uomo si costituisce nella coscienza inquieta di sé. Quando la scienza mostra sicurezza e razionalità la coscienza può aggiungere anche le proprie crisi e le ragioni dei conflitti storici. La biblioteca esibita da Petrarca mostra come l'io è il prodotto del sapere di sé attraverso il sapere acquisito. Il modo in cui la biblioteca filosofica e religiosa 
diventa parte integrante della costituzione intellettuale permette di fornire quelle risposte all'altezza della crisi. Un cristianesimo originario e inconsapevole non esiste più. Soltanto intellettualmente è possibile ricostituire una coscienza religiosa perché è stata una biblioteca a mettere in discussione il cristianesimo nella sua totalità storica e dottrinale. 